\title{
Endoplasmic Reticulum Stress Markers and Ubiquitin-Proteasome Pathway Activity in Response to a 200-km Run
}

\author{
HYO JEONG KIM ${ }^{1}$, CÉCILE JAMART ${ }^{2}$, LOUISE DELDICQUE ${ }^{2}$, GANG-LI AN ${ }^{2}$, YOON HEE LEE ${ }^{1}$, \\ CHANG KEUN KIM ${ }^{1}$, JEAN-MARC RAYMACKERS ${ }^{2}$, and MARC FRANCAUX ${ }^{2}$ \\ ${ }^{1}$ Human Physiology, Korea National Sport University, Seoul, SOUTH KOREA; and ${ }^{2}$ Institute of Neuroscience, \\ Université Catholique de Louvain, Louvain-la-Neuve, BELGIUM
}

\begin{abstract}
KIM, H. J., C. JAMART, L. DELDICQUE, G.-L. AN, Y. H. LEE, C. K. KIM, J.-M. RAYMACKERS, and M. FRANCAUX. Endoplasmic Reticulum Stress Markers and Ubiquitin-Proteasome Pathway Activity in Response to a 200-km Run. Med. Sci. Sports Exerc., Vol. 43, No. 1, pp. 18-25, 2011. Purpose: This study investigated whether a 200-km run modulates signaling pathways implicated in cellular stress in skeletal muscle, with special attention paid to the endoplasmic reticulum (ER) stress and to the activation of the ubiquitin-proteasome pathway. Methods: Eight men ran $200 \mathrm{~km}(28 \mathrm{~h} 03 \mathrm{~min} \pm 2 \mathrm{~h} 01 \mathrm{~min})$. Two muscle biopsies were obtained from the vastus lateralis muscle $2 \mathrm{wk}$ before and $3 \mathrm{~h}$ after the race. Mitogen-activated protein kinase, ubiquitin-proteasome pathway, ER stress, inflammation, and oxidative stress markers were assayed by Western blot analysis or by quantitative real-time polymerase chain reaction. Chymotrypsin-like activity of the proteasome was measured by a fluorimetric assay. Results: Phosphorylation states of extracellular signal-related kinase $1 / 2(+401 \% \pm 173.8 \%, P=0.027)$ and c-Jun $\mathrm{N}$-terminal $(+149 \% \pm 61.9 \%, P=0.023)$ increased after the race, whereas p38 phosphorylation remained unchanged. Increases in $\mathrm{BiP}(+235 \% \pm 94.7 \%, P=0.021)$ and in the messenger RNA level of total $(+138 \% \pm 31.2 \%, P=0.002)$ and spliced X-box binding protein $1(+241 \% \pm 53.3 \%, P=0.001)$ indicated the presence of ER stress. Transcripts of inflammatory markers interleukin- $6(+403 \% \pm 96.1 \%, P=0.002)$ and tumor necrosis factor $-\alpha(+233 \% \pm 58.4 \%, P=0.003)$ as well as oxidative stress markers metallothionein $1 \mathrm{~F}(+519 \% \pm 258.3 \%, P=0.042)$, metallothionein $1 \mathrm{H}(+666 \% \pm 157.5 \%, P=0.002)$, and nicotinamide adenine dinucleotide phosphate-oxidase (NADPH oxidase) $(+162 \% \pm 60.5 \%, P=0.016)$ were increased. The messenger RNA level of the ubiquitin ligases muscle-specific RING finger $1(+583 \% \pm 244.3 \%, P=0.024)$ and muscle atrophy F-box $(+249 \% \pm 83.8 \%, P=0.011)$ and the $\mathrm{C} 2$ proteasome subunit $(+116 \% \pm 40.6 \%, P=0.012)$ also increased. Surprisingly, the amount of ubiquitin-conjugated proteins and the chymotrypsin-like activity of the proteasome were decreased by $20 \% \pm 8.3 \%(P=0.025)$ and $21 \% \pm 4.4 \%(P=0.001)$, respectively. The expression of ubiquitin-specific protease 28 deubiquitinase was increased $(+81 \% \pm 37.9 \%$, $P=0.034)$. Conclusions: In the skeletal muscle, a $200-\mathrm{km}$ run activates the expression of ubiquitin ligases muscle-specific RING finger 1 and muscle atrophy F-box as well as various cellular stresses, among which are ER stress, oxidative stress, and inflammation. Meanwhile, compensatory mechanisms seem also triggered: the unfolded protein response is up-regulated, and the chymotrypsin-like activity of the proteasome is repressed. Key Words: ULTRAENDURANCE, BiP/GRP78, XBP1, INFLAMMATION, UBIQUITIN LIGASES, OXIDATIVE STRESS
\end{abstract}

$\mathrm{E}$ xtreme endurance exercise such as ultramarathons impairs metabolic state (4), increases oxidative stress $(14,33)$, and promotes an inflammatory state by enhancing cytokine production (22). Ultraendurance exercises also favor a catabolic state by activating protein degradation (6). In the skeletal muscle, an increase in the permeability of sarcoplasmic membrane has been reported (15) as well

Address for correspondence: Marc Francaux, Ph.D., Université Catholique de Louvain, Place Pierre de Coubertin - 1, B-1348 Louvain-la-Neuve, Belgium; E-mail: marc.francaux@uclouvain.be.

Submitted for publication January 2010.

Accepted for publication April 2010.

0195-9131/11/4301-0018/0

MEDICINE \& SCIENCE IN SPORTS \& EXERCISE

Copyright $\odot 2010$ by the American College of Sports Medicine

DOI: 10.1249/MSS.0b013e3181e4c5d1 as disorders in myofiber ultrastructure (3). The resulting disturbance of cell homeostasis gives the view of a general cellular stress, the causes of which being likely multiple.

A recently described cellular stress implicates the endoplasmic reticulum (ER), an organelle where folding and posttranslational modifications of proteins occur. ER is crucial in the selection and transport of proteins to other compartments both within and outside the cell. Certain stress conditions found at the level of skeletal muscle during ultraendurance exercise - for example, high cytosolic $\left[\mathrm{Ca}^{2+}\right]$, high levels of extracellular lipids, low glucose availability, or increased synthesis of secretory proteins - are known to disrupt ER homeostasis and to lead to the accumulation of unfolded or misfolded proteins within the ER lumen (9). To cope with this stress, cells activate a signal transduction system that links the ER lumen to the cytoplasm and the nucleus; this process is called the unfolded protein response (UPR; for a recent review, see Ron and Walter [32]). The 
UPR is required for the restoration of normal ER function through three main actuators: activating transcription factor 6 (ATF6), inositol-requiring enzyme $1 \alpha$ (IRE1 $\alpha$ ), and protein kinase R-like ER protein kinase (PERK). In their inactive state, each of these factors associates with the chaperone protein $\mathrm{BiP}$ (binding protein) also called glucoseregulated protein 78 , a member of the heat shock protein 70 (Hsp70) family. On accumulation of unfolded/misfolded proteins in the ER lumen, ATF6, IRE1 $\alpha$, and PERK are released from $\mathrm{BiP}$ and become activated. The downstream effectors of these three pathways induce the expression of genes, such as X-box binding protein 1 (XBP1), CCAAT/ enhancer binding protein (C/EBP) homologous protein $(C H O P)$, and activating transcription factor 4 (ATF4), which encode proteins that function to augment the ER protein-folding capacity. The UPR leads to the expression of molecular chaperones, the most studied being BiP, calnexin, protein disulfide isomerase (PDI), and ER-residing protein endoplasmic oxidoreductin $1 \alpha($ ERO1- $\alpha)$, which associate transiently with numerous newly synthesized proteins during their maturation in the ER. The presence of chaperones in the ER membrane alleviates the stress by up-regulating appropriate folding and assembly of new proteins (32).

The UPR also aims to reduce cellular stress by inhibiting protein synthesis and by activating protein degradation. PERK activation leads to the phosphorylation of eukaryotic initiation factor $2 \alpha$, which inhibits the assembly of the $80 \mathrm{~S}$ ribosome and results in a general inhibition of protein synthesis. ER-associated protein degradation is a mechanism by which proteins in the ER are exported to the cytoplasm for destruction by the proteasome (36). After an increase in chaperone synthesis and translational attenuation, the activation of protein degradation represents a third mechanism used by UPR to cope with ER stress.

Muscle protein degradation is a complex process implicating four systems: the lysosomal proteases, the $\mathrm{Ca}^{2+}$. dependent proteases, the caspases, and the ubiquitinproteasome pathways (UPP); the latter can be activated by ER-associated protein degradation as mentioned previously. The contribution of each mechanism to muscle protein degradation during ultraendurance exercise is unknown, but UPP is likely to be the major actuator because it can degrade the bulk of intracellular proteins, including myofibrillar proteins (30).

We had the opportunity to take, in field conditions, muscle biopsies in runners engaged in a $200-\mathrm{km}$ run. The purposes of this study were to examine different cellular stresses potentially triggered during an ultraendurance exercise and, more specifically, to describe: 1) the presence of ER stress in skeletal muscle because, to the best of our knowledge, activation of ER stress has never been described during exercise; 2) the activation of UPP in skeletal muscle; 3 ) the existence of an oxidative stress and an inflammatory response in muscle cells; and 4) the activation of stresssensitive signaling cascades, namely, the mitogen-activated protein kinase (MAPK) pathways.

\section{METHODS}

Protocol. Eight men, whose characteristics are presented in Table 1, took part in a $200-\mathrm{km}$ running race held at sea level in Cheju Island (South Korea). They were experienced and well-prepared ultramarathon runners so that all completed the distance within the cutoff time of $36 \mathrm{~h}$. The local temperature ranged from $12.5^{\circ} \mathrm{C}$ to $25.3^{\circ} \mathrm{C}$ (mean $=15.9^{\circ} \mathrm{C}$ ) with a relative humidity between $59.0 \%$ and $63.4 \%$ and a wind speed of $3-4.5 \mathrm{~km} \cdot \mathrm{h}^{-1}$. The experimental protocol used in this study was approved by the ethical committee of the Korea National Sport University, and all the procedures used were in accordance with the Declaration of Helsinki. The subjects were completely informed about the experimental protocol before their written consent was obtained.

Two weeks before the race, the subjects reported to the laboratory in the morning. They were refrained from exercise for a week before obtaining muscle sample and fed their usual breakfast $3 \mathrm{~h}$ before the biopsy. A biopsy sample was taken from the mid portion of the vastus lateralis muscle with a 5-mm Bergström biopsy needle after local skin anesthesia with $2 \%$ lidocaine. The samples were immediately frozen in liquid nitrogen and stored at $-80^{\circ} \mathrm{C}$ for later analysis. During the race and the recovery, the runners were provided with food and drink ad libitum. Three hours after race completion, a second muscle biopsy was taken about $1 \mathrm{~cm}$ apart from the first biopsy site in the same leg and following the same procedure.

RNA extraction and quantitative real-time polymerase chain reaction. Tissue samples $(\sim 30 \mathrm{mg})$ were homogenized in $1 \mathrm{~mL}$ of TRIzol reagent (Invitrogen, Vilvoorde, Belgium), and RNA was isolated according to the manufacturer's instructions. RNA quality and quantity were assessed by $1.5 \%$ agarose gel electrophoresis and NanoDrop spectrophotometry. Reverse transcription was performed on MyIQ2 thermocycler (Bio-Rad, Nazareth, Belgium). $1.5 \mu \mathrm{g}$ of RNA was associated with $10 \mu \mathrm{L}$ of reverse transcription (RT) buffer, $1 \mu \mathrm{L}$ of $20 \times$ Enzyme Mix (Bio-Rad), and RNase-free water to a final volume of $20 \mu \mathrm{L}$ that was run for $60 \mathrm{~min}$ at $37^{\circ} \mathrm{C}$ followed by $5 \mathrm{~min}$ at $95^{\circ} \mathrm{C}$. Primers used for quantitative polymerase chain reaction are reported in Table 2. Primers of the total form of XBP1 (XBP1t) were able to recognize both the spliced and the unspliced forms of XBP1 messenger RNA (mRNA), whereas XBP1s primers were designed to amplify only the spliced form. NADPH

TABLE 1. Subject characteristics and performance.

\begin{tabular}{lcccc}
\hline Subject & Age $\mathbf{( y r )}$ & Height $\mathbf{( c m )}$ & Weight $\mathbf{( k g )}$ & Race Time (h:min) \\
\hline 1 & 46 & 168 & 66.2 & $22: 53$ \\
2 & 41 & 178 & 80.4 & $22: 53$ \\
3 & 40 & 170 & 68.2 & $22: 15$ \\
4 & 38 & 171 & 69.5 & $23: 32$ \\
5 & 44 & 172 & 63.3 & $31: 36$ \\
6 & 48 & 172 & 71.8 & $31: 08$ \\
7 & 45 & 164 & 66.9 & $35: 04$ \\
8 & 50 & 165 & 74.2 & $35: 04$ \\
Mean & 44 & 170 & 70.1 & $28: 03$ \\
SEM & 1 & 2 & 1.9 & $2: 01$
\end{tabular}


TABLE 2. Sequences of primers $\left(5^{\prime}-3^{\prime}\right)$.

\begin{tabular}{lcc}
\hline & \multicolumn{1}{c}{ Forward } & Reverse \\
\hline ATF4 & CCA ACA ACA GCA AGG AGG ATG & GTC ATC CAA CGT GGT CAG AAG G \\
CHOP & CTG GCT TGG CTG ACT GAG GAG & CGG GCT GGG GAA TGA CC \\
C2 & CAT TGA AAA GGG CGC AAT C & GCC ATA TCG TTG TGT TGG TA \\
IL-6 & TGG ATT CAA TGA GGA GAC TTG & GAT TCT TTG CCT TTT TCT GC \\
MAFbx & CGA CCT CAG CAG TTA CTG CAA C & TTT GCT ATC AGC TCC AAC AG \\
MT1F & CCT CCC CTG ACT ATC AAA GCA & GCA CTT GCA GGA ACC AGC \\
MT1H & TGT TCC ACT GCC TCT TCT CTT C & CAG CTC TTC TTG CAG GAG GTG \\
MuRF-1 & AAA CAG GAG TGC TCC AGT CGG & CGC CAC CAG CAT GGA GAT ACA \\
NADPHox & TGG CTG CCC ATC TGG TGA ATG & CAG CAG CCC TCC TGA AAC ATG C \\
TNF- $\alpha$ & GGT GCT TGT TCC TCA GCC TCT & AGG GTT TGC TAC AAC ATG GGC \\
USP28 & TGC CTT CTG GAG CTG AAT G & CCA AGG TAA GAG CAC CAA TG \\
XBP1s & CGC TTG GGG ATG GAT GC & TGG GCC TGC ACC TGC TG \\
XBP1t & CGC TTG GGG ATG GAT GC & TGG GGA GAT GTT CTG GAG GG \\
$\beta 2 M G$ & ATG AGT ATG CCT GCC GTG TGA & GGC ATC TTC AAA CCT CCA TG \\
\hline
\end{tabular}

oxidase (NADPHox) primers were designed to target the catalytic Nox4 subunit. $\beta$-2-microglobulin $(\beta 2 M G)$ was used as reference gene because it was previously shown to be unaffected by exercise (5). Our data confirmed that $\beta 2 M G$ was unchanged after the $200-\mathrm{km}$ run. Experiments were performed on a MyIQ2 thermocycler using the following conditions: $3 \mathrm{~min}$ at $95^{\circ} \mathrm{C}$, followed by 35 cycles of $30 \mathrm{~s}$ at $95^{\circ} \mathrm{C}, 30 \mathrm{~s}$ at $60^{\circ} \mathrm{C}$, and $30 \mathrm{~s}$ at $72^{\circ} \mathrm{C}$. Samples were analyzed in triplicate in $25.5 \mu \mathrm{L}$ of reaction volume containing $12.5 \mu \mathrm{L}$ of IQ SybrGreen SuperMix (Bio-Rad), $0.25 \mu \mathrm{L}$ of each primer (final concentration $=100 \mathrm{nM}$ ), and $12 \mu \mathrm{L}$ of complementary DNA. Melting curves were systematically analyzed to ensure the specificity of the amplification process.

Protein extraction. About $10-20 \mathrm{mg}$ of frozen tissue was ground in a mortar and homogenized in an icecold buffer (20 mM Tris, $\mathrm{pH} 7.0,270 \mathrm{mM}$ sucrose, $5 \mathrm{mM}$ EGTA, $1 \mathrm{mM}$ EDTA, 1\% Triton X-100, $1 \mathrm{mM}$ sodium orthovanadate, $50 \mathrm{mM}$ sodium $\beta$-glycerophosphate, $5 \mathrm{mM}$ sodium pyrophosphate, $50 \mathrm{mM}$ sodium fluoride, $1 \mathrm{mM}$ 1,4-dithiothreitol, and a protease inhibitor cocktail containing $1 \mathrm{mM}$ EDTA (Roche Applied Science, Vilvoorde, Belgium)) for $5 \mathrm{~min}$ on ice. The homogenates were then centrifuged for $10 \mathrm{~min}$ at $10,000 \mathrm{~g}$. The supernatants were immediately stored at $-80^{\circ} \mathrm{C}$. Protein concentration was determined using the DC protein assay kit (Bio-Rad).

Western blot analysis. Cell lysates (50-80 $\mu \mathrm{g}$ of protein) were combined with Laemmli sample buffer and separated by sodium dodecyl sulfate-polyacrylamide gel electrophoresis. For protein carbonyl measurements, $5 \mu \mathrm{g}$ of protein was derivatized with 2,4-dinitrophenyl hydrazine before electrophoresis following instructions given by the protein oxidation kit from Chemicon (Brussels, Belgium). After electrophoretic separation at $40 \mathrm{~mA}$, the proteins were transferred to a polyvinylidene fluoride membrane at $80 \mathrm{~V}$ for $2 \mathrm{~h}$ for a Western blot analysis. Membranes were then incubated in a Blotto solution $(5 \%$ nonfat milk in Tris-buffered saline with $0.1 \%$ Tween 20 (TBST).). Subsequently, membranes were incubated with one of the following antibodies overnight at $4^{\circ} \mathrm{C}$ : phospho-p38 (p38 protein kinase) Thr 180/Tyr 182, p38 total, phospho-extracellular signal-related kinase 1/2 (ERK1/2) Thr 202/Tyr 204, ERK1/2 total, phospho-c-Jun N-terminal (JNK) Thr 183/
Tyr 185, JNK total, dinitrophenyl, inhibitor of nuclear factor $\kappa \mathrm{B} \alpha(\mathrm{I} \kappa \mathrm{B} \alpha)$ total, $\mathrm{BiP}$, calnexin, ERO1- $\alpha$, PDI, ubiquitin, proteasome 20S $\alpha+\beta$, and glyceraldehyde 3-phosphate dehydrogenase (GAPDH). All antibodies were from Cell Signaling (Leiden, The Netherlands), except proteasome 20S $\alpha+\beta$ and GAPDH from Abcam (Cambridge, United Kingdom) and dinitrophenyl from Sigma (Bornem, Belgium).

Membranes were washed in TBST and incubated for $1 \mathrm{~h}$ at room temperature in a secondary antibody conjugated to horseradish peroxidase (1:10,000; Cell Signaling). After an additional three washes, chemiluminescence detection was carried out using an enhanced chemiluminescent Western blotting kit (ECL Plus; Amersham Biosciences, Diegem, Belgium). The films were then scanned on an ImageScanner using the Labscan software and quantified with the Image Master 1D Image Analysis Software (Amersham Biosciences). Results were reported with GAPDH for adjusting unequal protein loading or with the total form of the protein when the phosphorylation state was studied.

Proteasome activity. Chymotrypsin-like activity of the $20 \mathrm{~S}$ proteasome was assayed by the release of fluorescent 7-amino 4-methylcoumarin (AMC) at $37^{\circ} \mathrm{C}$, on a 96-well black plate (Greiner bio-one, Wemmel, Belgium). In each well, $100 \mu \mathrm{M}$ Suc-LLVY-AMC (Merck, Darmstadt, Germany) and $200 \mu \mathrm{L}$ of buffer (50 mM Tris- $\mathrm{HCl} \mathrm{pH} 8$, $5 \mathrm{mM} \mathrm{MgCl}_{2}, 1 \mathrm{mM}$ 1,4-dithiothreitol) were added to $50 \mu \mathrm{g}$ of protein extract. Each sample was analyzed in triplicate, with one replicate in which $50 \mu \mathrm{M}$ MG-132 (Merck), a proteasome inhibitor, was added to assess the specificity of the activity. Release of AMC was measured every 2 min for 30 min on a fluorometer (Fluoroskan Ascent FL; Thermo Scientific, Zellik, Belgium), at an excitation and emission wavelength of 370 and $460 \mathrm{~nm}$, respectively. Enzymatic activity was calculated as the slope of the accumulation of fluorescence, as a function of time, and was normalized to the amount of $20 \mathrm{~S} \alpha+\beta$ proteasome protein measured by Western blot analysis.

Statistics. The results after exercise are presented in percentage of changes versus the preexercise condition (mean $\pm \mathrm{SEM}$ ). The statistical significance of these changes was estimated by paired $t$-test. The threshold of significance was set at $P<0.05$.

\section{RESULTS}

The fastest runner completed the 200-km ultramarathon within $22.2 \mathrm{~h}\left(9.0 \mathrm{~km} \cdot \mathrm{h}^{-1}\right)$, whereas the slowest one needed $35 \mathrm{~h}$ to achieve the same distance $\left(5.7 \mathrm{~km} \cdot \mathrm{h}^{-1}\right)$. Muscle biopsies were taken $3 \mathrm{~h}$ after the arrival of each subject. At that time, p38 phosphorylation state was unchanged, whereas the phosphorylation states of ERK1/2 and JNK were increased by $401 \% \pm 173.8 \%(P=0.027)$ and by $149 \% \pm$ $61.9 \%(P=0.023)$, respectively (Fig. 1$)$.

mRNA levels of markers of oxidative stress, i.e., metallothionein 1F (MT1F), metallothionein 1H (MT1H), and NADPHox, were increased by $519 \% \pm 258.3 \%(P=0.042)$, 

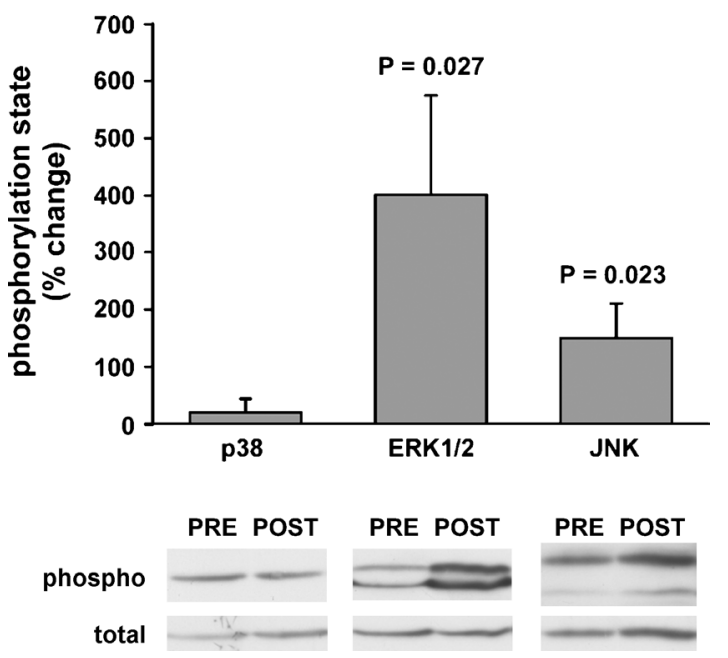

FIGURE 1-Changes in phosphorylation states of MAPK after a 200-km run (p38 (Thr 180/Tyr 182), ERK1/2 (Thr 202/Tyr 204), JNK (Thr 183/Tyr 185)). Percentage of change: mean \pm SEM. Representative Western blots for the phosphorylated and total forms are added.

$666 \% \pm 157.5 \%(P=0.002)$, and $162 \% \pm 60.5 \%(P=0.016)$, respectively, after the race (Fig. 2A). Protein carbonyls were unchanged (Fig. 2B). Interleukin-6 (IL-6) mRNA level increased by $403 \% \pm 96.1 \%(P=0.002)$ and tumor necrosis factor- $\alpha(\mathrm{TNF}-\alpha)$ by $233 \% \pm 58.4 \%(P=0.003$; Fig. $3 \mathrm{~A})$. $\mathrm{I} \kappa \mathrm{B} \alpha$ protein level showed a large interindividual variability.
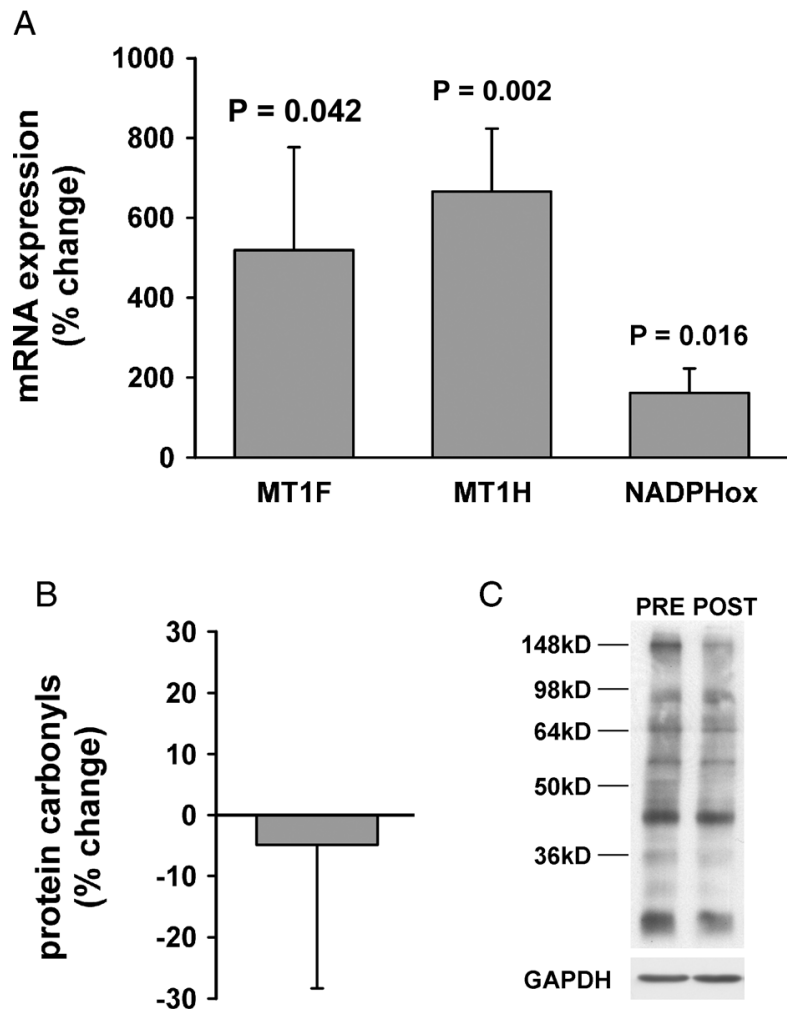

FIGURE 2-Changes in oxidative stress markers after a 200-km run. Percentage of change: mean \pm SEM. A, mRNA level of MT1F, MT1H, and NADPHox. B, Protein carbonyl level. C, Representative Western blot with control for loading.
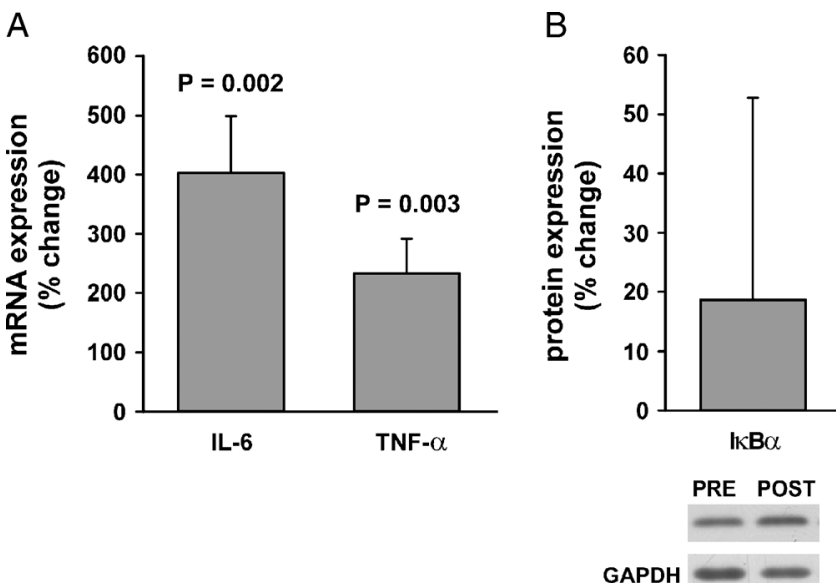

FIGURE 3-Changes in inflammation markers after a 200-km run. Percentage of change: mean \pm SEM. A, mRNA level of IL-6 and TNF- $\alpha$. $B$, Protein expression of $I \kappa B \alpha$ total. A representative Western blot with control for loading is added.

As a consequence, the mean value was not altered in comparison with the preexercise value (Fig. 3B).

Transcription of XBP1 is a consequence of ER stress and is regulated by the ATF6 pathway. The level of XBP1t mRNA was increased by $138 \% \pm 31.2 \%(P=0.002)$, and XBP1s was increased by $241 \% \pm 53.3 \%(P=0.001$;
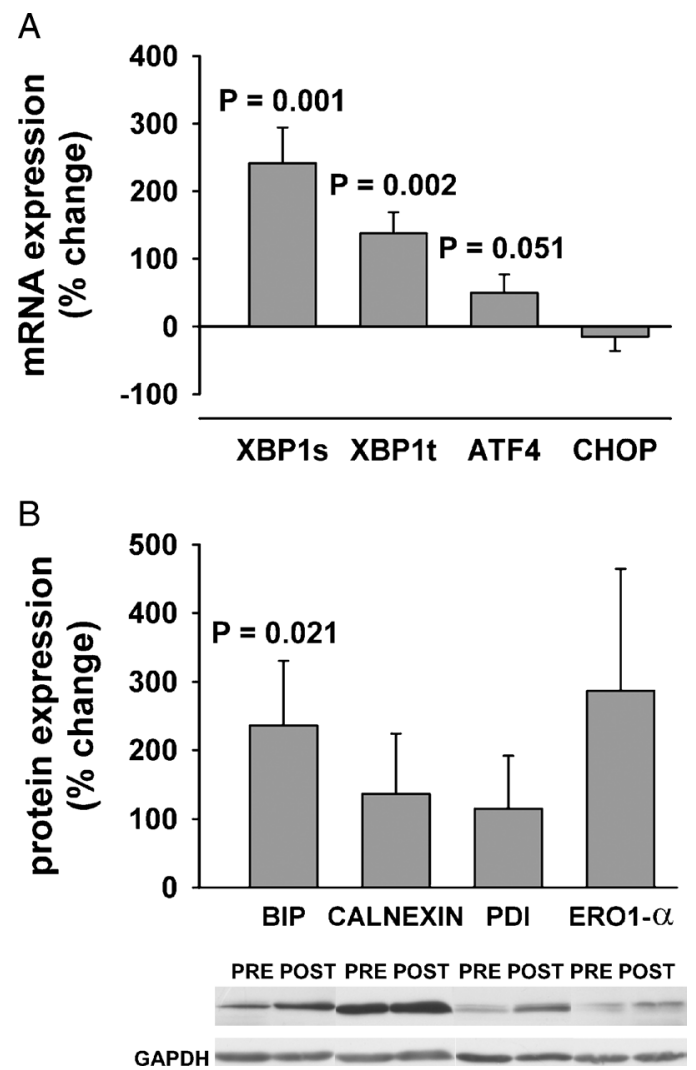

FIGURE 4-Changes in ER stress markers after a 200-km run. Percentage of change: mean \pm SEM. A, mRNA levels of XBP1s, XBP1t, ATF4, and CHOP. B, Protein expression of BiP, also called GRP78, PDI, and ERO1- $\alpha$. A representative Western blot with control for loading is added. 

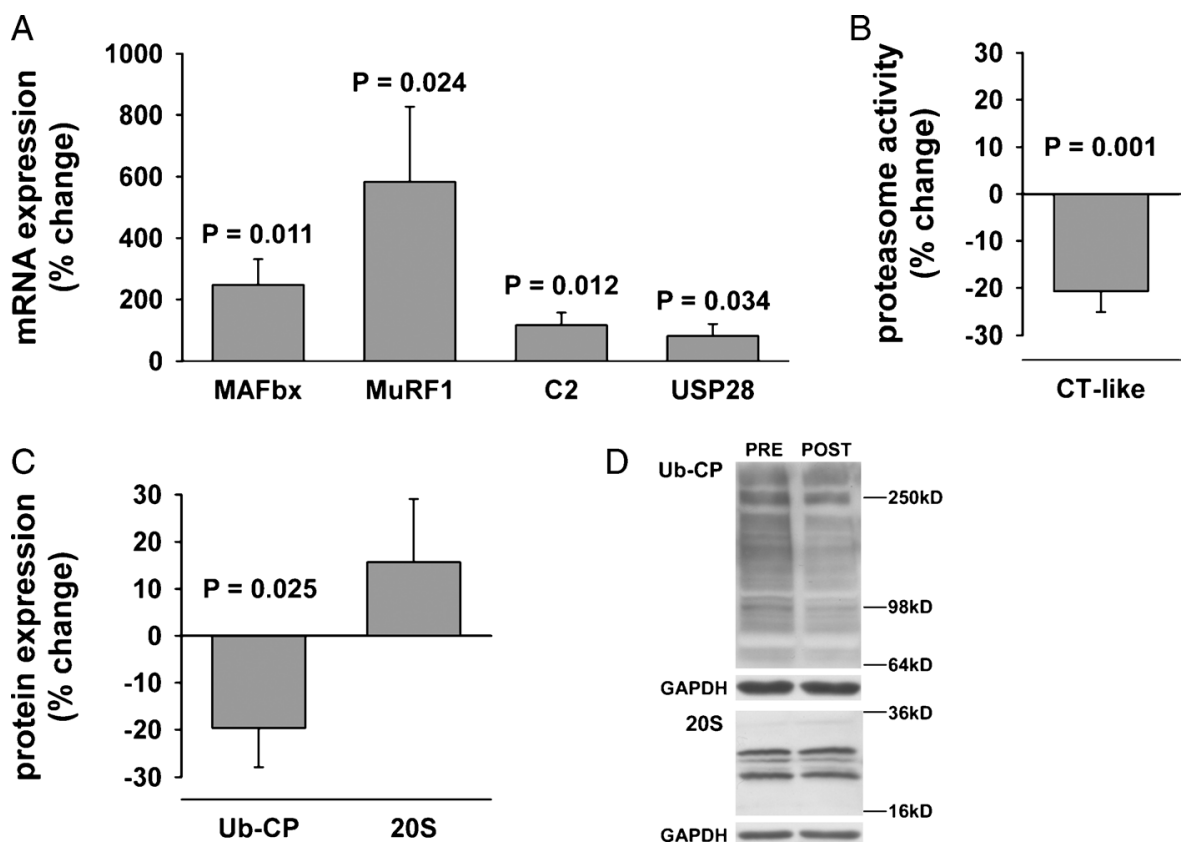

FIGURE 5-Changes in UPP after a 200-km run. Percentage of change: mean \pm SEM. A, mRNA level of MAFbx, MuRF-1, C2, and USP28. B, Chymotrypsin-like activity of the proteasome (CT-like). C, Level of UbCP and protein expression of 20S. D, Representative Western blots with control for loading.

Fig. 4A). This suggests a regulation on the transcription of the gene and the splicing of its transcript, the latter being under the control of the IRE1 $\alpha$ pathway. On the other hand, mRNA levels of ATF4 and CHOP, two other genes implicated in the response to ER stress and regulated by the PERK pathway, did not change (Fig. 4A). The expression of BiP protein increased by $235 \% \pm 94.7 \%(P=0.021)$, whereas other chaperone proteins known to reflect ER stress did not change significantly (Fig. 4B). Large variability between subjects was observed in the expression of those proteins. In basal conditions, ERO1- $\alpha$ was undetectable in two subjects. Consequently, only results for six subjects are reported for that protein (Fig. 4B).

The mRNA level of muscle-specific ubiquitin ligases muscle-specific RING finger 1 (MuRF-1) and muscle atrophy F-box (MAFbx; also known as atrogin-1) was increased by $583 \% \pm 244.3 \%(P=0.024)$ and $249 \% \pm 83.8 \%$ $(P=0.011)$, respectively (Fig. 5A). Similarly, the mRNA of the $\mathrm{C} 2$ proteasome subunit was increased by $116 \% \pm 40.6 \%$ $(P=0.012$, Fig. 5A). Increases in the expression of genes coding for ubiquitin ligases and for a subunit of proteasome are elements in favor of a positive regulation of the UPP after exercise. Therefore, it was unexpected to observe that the chymotrypsin-like activity of the proteasome was reduced by $21 \% \pm 4.4 \%(P=0.001$; Fig. $5 \mathrm{~B})$ and that the level of ubiquitin-conjugated proteins (UbCP) diminished by $20 \% \pm$ $8.3 \%(P=0.025$; Figs. 5C and D) after the race, whereas the expression of the $20 \mathrm{~S} \alpha+\beta$ proteasome subunit was unchanged (Figs. 5C and D). These observations suggest antagonistic modes of regulation of the ubiquitin-proteasome system during a long-lasting exercise. Deubiquitinating en- zymes could be implicated in these antagonistic modes of regulation as suggested by the increase in the mRNA level of ubiquitin-specific protease 28 (USP28; $+81 \% \pm 37.9 \%$, $P=0.034$; Fig. 5A).

\section{DISCUSSION}

Only a few studies have analyzed the role of ER stress in the skeletal muscle $(25,28)$. In the earliest publication, ER stress markers were reported to be unaltered in the skeletal muscle when obesity and high-fat diet were used as cellular stress inducers in animals. However, the ER stress markers used to support the assertion were not specified in that publication (25). A very recent study in diabetic patients indicated that markers of the UPR were increased in myogenic cells isolated from muscle biopsies and cultured in the presence of palmitate, indicating that ER stress can be elicited in human muscle cells (28). The main finding of the present study is to show, for the first time, the presence of ER stress markers in the human skeletal muscle after an ultraendurance exercise. This is compatible with the view that exercise causes brief and sometimes robust cellular stresses that are often necessary for inducing muscle adaptation.

XBP1s mRNA is known to be a highly specific marker of ER stress (8). Transcription of XBP1 is up-regulated by the translocation of the cytoplasmic domain of ATF6 to the nucleus. When activated, IRE $1 \alpha$ catalyzes the removal of a 26-nucleotide intron from XBP1, RNA creating the spliced form that encodes for an active transcription factor. Our results suggest that ultraendurance exercise activates the 
ATF6 and IRE1 $\alpha$ pathways because both XBP1t and XBP1s were increased in the muscle samples. In this study, we were not able to show any implication of the third signaling cascade activated by ER stress, namely, the PERK pathway, in response to ultraendurance exercise as the expression of ATF4 and CHOP, two genes regulated by this pathway, was not changed $3 \mathrm{~h}$ after the race. Further research is required to shed light on the mechanisms by which ultraendurance exercise is able to specifically activate two cascades of the UPR.

Oxidative stress is known to trigger ER stress because reactive oxygen species (ROS) are able to directly damage proteins in the ER folding machinery and to induce an accumulation of misfolded protein, leading to the UPR activation (17). The ER seems to be particularly sensitive to the redox state of the cell because it contains proteins with a high oxidizing potential specialized in protein folding and disulfide bond formation. Alternatively, ROS may cause ER stress via generation and accumulation of oxidized proteins (35). In the skeletal muscle, the generation of ROS is low at rest. During exercise, the skeletal muscle produces much superoxide anions because of its higher oxygen uptake. A recent article showed that mitochondrial $\mathrm{H}_{2} \mathrm{O}_{2}$ was increased during an ultraendurance event (33). Muscle damage occurring during an ultramarathon activates inflammatory processes (11), which may also play an important role in ROS production. In the present study, ROS production was not measured, but we show that NADPHox, MT1F, and MT1H mRNA were increased after the race. Chronic oxidative stress is known to be accompanied by up-regulation of NAD(P)H oxidase (31), and exercise-induced oxidative stress has been showed to trigger the expression of the antioxidants factors MT1F and MT1H $(13,27)$. Three hours after exercise completion, the protein carbonyl level was unaltered in comparison with basal conditions. This observation is compatible with the findings of Sahlin et al. (33) who showed that ROS production was restored to basal level at recovery of an ultraendurance event. This suggests that the carbonyls could be quickly removed after the end of the exercise. The causal relationship between ROS production and ER stress during exercise remains to be demonstrated.

$\mathrm{BiP}$ is the most abundant protein in the ER lumen, and its expression is regulated by the UPR (40). The larger amount of BiP observed in the muscle after the ultramarathon may be interpreted as an adaptive mechanism by which the skeletal muscle protects itself against subsequent ER stress. Nevertheless, other chaperones analyzed in the present study were not changed significantly $3 \mathrm{~h}$ after the race possibly because the kinetics of expression is slower, because repetitive stimulations are required, or because they are not affected by the ultraendurance exercise.

Exercise has been reported to modulate UPP activation in the human skeletal muscle depending on the loading modality and periods (19). UPP works in a two-step sequence. First, the substrate protein is conjugated to a polyubiquitin chain that leads to the formation and the accumulation of $\mathrm{UbCP}$ (30). In this process, ligases are responsible for the specific recognition of the numerous substrates of the UPP and seem to be the key regulators of the pathway. Transcriptome analyses have revealed muscle-specific ligases among which are MAFbx and MuRF-1 (1), which interact with and possibly ubiquitinate a variety of myofibrillar proteins (38). Here, we show that mRNA for MuRF-1 and MAFbx was increased after the ultramarathon. The transcriptional regulation of MuRF-1 and MAFbx is controlled by the members of the forkhead Foxo family. These transcription factors are inactivated by Akt (also known as protein kinase $\mathrm{B}$ ). The phosphorylation state of Akt (Ser-473) was unchanged $3 \mathrm{~h}$ after the race in comparison with basal conditions (data not shown). Akt is highly sensitive to insulin and dietary state $(2,39)$. We may not rule out that the activity of Akt was not decreased during the race because the runners were fed between the end of the race and the time of sampling biopsy. The levels of MuRF-1 and MAFbx mRNA are also related to ROS production and proinflammatory cytokines (18). The higher levels of NADPHox, MT1F, MT1H, and TNF- $\alpha$ mRNA after the race suggest that signaling events involving oxidative stress and inflammation may also be implicated either in a transcriptional regulation or in a higher stability of MuRF-1 and MAFbx.

The second step of the UPP is the degradation of the $\mathrm{UbCP}$ by the $26 \mathrm{~S}$ proteasome, a large multicatalytic protease complex comprising a $20 \mathrm{~S}$ catalytic core, which contains the proteolytic sites, and a $19 \mathrm{~S}$ regulatory complex, which is responsible for substrate recognition and unfolding. The 20S proteasome exhibits chymotrypsin-like, trypsin-like, and caspase-like catalytic activities, the first one being the most important in the skeletal muscle (7). The increases that we observed in the expression of genes coding for ubiquitin ligases and for a subunit of proteasome are favorable elements for the positive regulation of the UPP and for the activation of protein breakdown. Surprisingly, however, the amount of $\mathrm{UbCP}$ and the chymotrypsin-like activity of the proteasome were not only lower after the race than that in basal conditions, but no change in the protein level of the $20 \mathrm{~S} \alpha+\beta$ proteasome subunits was detectable. These observations suggest antagonistic modes of regulation of the ubiquitinproteasome system in response to ultraendurance exercise. Deubiquitinating enzymes could be implicated in these antagonistic modes of regulation as suggested by the increase in the mRNA level of USP28, which is a member of the ubiquitin-specific proteases. The function of this group of enzymes is to reverse the ubiquitinating reaction by removing ubiquitin moiety from a large variety of substrates. The increase in the muscle-specific E3 ligases, MuRF-1 and MAFbx, and in USP28 suggests that the ubiquitination/ deubiquitination cycle could be enhanced by muscle cells in response to ultraendurance exercise. This is not incompatible with an increased UPP activity because deubiquitinating enzymes not only prevent inappropriate proteasomal degradation but also take a role in polyubiquitin chain recycling after proteasomal degradation (37). 
The proteasome $\mathrm{C} 2$ is one of the $20 \mathrm{~S}$ proteasome subunits, and it seems to be important for the stimulation of the peptidase activities of 20S. C2 mRNA was increased during the race, suggesting a transcriptional regulation induced by endurance exercise. However, no change in the protein level of the 20S $\alpha+\beta$ proteasome subunits was detectable, and unexpectedly, the chymotrypsin-like activity of the proteasome was decreased. The activity measured in vitro by chemical assays provides a catalytic activity of the $20 \mathrm{~S}$ subunit of the proteasome for a determined substrate concentration. Our data suggest the presence of an inhibitory mechanism that would down-regulate the chymotrypsin-like activity of the proteasome in response to ultraendurance exercise. Although the mechanism of regulation is unknown, oxidative stress is a potential candidate because it has been shown to repress the chymotrypsin-like activity of proteasome in vitro (10).

Solid evidence supports an up-regulation of IL-6 by exercise both at the mRNA and at the protein levels (for review, see Nielsen and Pedersen [20]). Our results are compatible to those previous findings. The increase in IL-6 mRNA was more pronounced in the four slowest runners than in the four fastest runner ( $t$-test, $P=0.046)$, suggesting that the duration of the exercise instead of the intensity is the main regulator of IL- 6 expression in response to ultraendurance exercise. IL-6 has proinflammatory and anti-inflammatory properties (29) and has been showed to suppress TNF- $\alpha$ production in exercising humans (34). After resistance exercise, both IL- 6 and TNF- $\alpha$ mRNA are increased in the skeletal muscle, but plasma concentrations are only modestly altered (21). This highlights the importance of IL-6 plasma concentration, which is probably large enough to blunt TNF- $\alpha$ expression in the skeletal muscle during endurance events such as marathons (24) but not during resistance exercise (21). In this study, we observed a clear increase in TNF- $\alpha$ mRNA after the $200-\mathrm{km}$ run despite a large increase in plasma IL-6 concentration (11). Although we were not able to show any decrease in the I $\kappa \mathrm{B} \alpha$ level, we may not rule out that the nuclear factor $\kappa \mathrm{B}$ pathway was activated during exercise and rapidly inactivated at recovery. Taken together, our observations support the idea that an ultramarathon induces a strong proinflammatory stress in the skeletal muscle as assessed previously at the systemic level $(23,26)$. Nevertheless, immune cells are known to invade damaged muscles, and therefore, we may not rule out any contribution of inflammatory cells in the TNF- $\alpha$ mRNA increase that we observed.

MAPK activity is triggered by various cellular stressors such as ROS production, acidification, mechanical perturbations, glycogen depletion, and high cytosolic $\mathrm{Ca}^{2+}$ concentration (for review, see Koulmann and Bigard [12]). Several reports provide evidence that JNK, ERK1/2, and p38 MAPK are activated during endurance exercise (12). The specific activation of each member of the MAPK family seems dependent on the duration and the intensity of exercise, the modalities of muscle contractions, and the time of muscle sampling (12). The results of the present study confirm that the MAPK pathways are activated in response to ultraendurance exercise because the phosphorylation states of $\mathrm{JNK}$ and ERK1/2 were increased. Because p38 is known to be rapidly dephosphorylated after completion of the exercise (16), it is likely that we missed its activation by taking the biopsies $3 \mathrm{~h}$ after the end of the race.

The present study is descriptive. That is its main limitation. Neither the exercise intensity nor the food intake was controlled, and there is no group with unexercised subjects. The muscle samples were taken $3 \mathrm{~h}$ after completion of the race. This timing brings various unavoidable limitations in the interpretation of the results. Nevertheless, our field observations provide novel results acquired on human muscle samples regarding the cellular stress induced by an extremely long exercise protocol. The present data show for the first time that ultraendurance exercise activates ER stress; moreover, they extend previous observations made during endurance exercise that the stress-related MAPK pathways, oxidative stress and inflammation, are activated; finally, they shed light on unexpected regulation of the UPP, namely, an up-regulation of E3 ligases concomitant with a decrease in the $20 \mathrm{~S}$ activity of proteasome.

Louise Deldicque is a postdoctoral researcher from the Fonds de la Recherche Scientifique FRS-FNRS. Gang-Li An is supported by the Association Belge contre les Maladies Neuro-Musculaires and the Fonds Spéciaux de Recherche from the Université Catholique de Louvain. This study was funded by Fonds de la Recherche Scientifique Médicale (FRSM 3.4514) and by a research grant from the Korea National Sport University.

The authors did not receive funding for this work from any of the following organizations: National Institutes of Health, Wellcome Trust, Howard Hughes Medical Institute, and other(s).

The results of the present study do not constitute endorsement by the American College of Sports Medicine.

\section{REFERENCES}

1. Bodine SC, Latres E, Baumhueter S, et al. Identification of ubiquitin ligases required for skeletal muscle atrophy. Science. 2001;294(5547):1704-8.

2. Brozinick JT Jr, Birnbaum MJ. Insulin, but not contraction, activates Akt/PKB in isolated rat skeletal muscle. J Biol Chem. 1998; 273(24):14679-82.

3. Crenshaw AG, Friden J, Hargens AR, Lang GH, Thornell LE. Increased technetium uptake is not equivalent to muscle necrosis: scintigraphic, morphological and intramuscular pressure analyses

of sore muscles after exercise. Acta Physiol Scand. 1993;148(2): 187-98.

4. Davies CT, Thompson MW. Physiological responses to prolonged exercise in ultramarathon athletes. J Appl Physiol. 1986;61(2): 611-7.

5. Deldicque L, Atherton P, Patel R, et al. Effects of resistance exercise with and without creatine supplementation on gene expression and cell signaling in human skeletal muscle. $J$ Appl Physiol. 2008;104(2):371-8. 
6. Dohm GL, Tapscott EB, Kasperek GJ. Protein degradation during endurance exercise and recovery. Med Sci Sports Exerc. 1987; 19(5 suppl):S166-71.

7. Farout L, Lamare MC, Cardozo C, Harrisson M, Briand Y, Briand M. Distribution of proteasomes and of the five proteolytic activities in rat tissues. Arch Biochem Biophys. 2000;374(2): 207-12.

8. Hirota M, Kitagaki M, Itagaki H, Aiba S. Quantitative measurement of spliced XBP1 mRNA as an indicator of endoplasmic reticulum stress. J Toxicol Sci. 2006;31(2):149-56.

9. Huang G, Yao J, Zeng W, et al. ER stress disrupts $\mathrm{Ca}^{2+}$-signaling complexes and $\mathrm{Ca}^{2+}$ regulation in secretory and muscle cells from PERK-knockout mice. J Cell Sci. 2006;119(pt 1):153-61.

10. Ishii T, Sakurai T, Usami H, Uchida K. Oxidative modification of proteasome: identification of an oxidation-sensitive subunit in $26 \mathrm{~S}$ proteasome. Biochemistry. 2005;44(42):13893-901.

11. Kim HJ, Lee YH, Kim CK. Biomarkers of muscle and cartilage damage and inflammation during a $200 \mathrm{~km}$ run. Eur J Appl Physiol. 2007;99(4):443-7.

12. Koulmann N, Bigard AX. Interaction between signalling pathways involved in skeletal muscle responses to endurance exercise. Pflugers Arch. 2006;452(2):125-39.

13. Mahoney DJ, Parise G, Melov S, Safdar A, Tarnopolsky MA. Analysis of global mRNA expression in human skeletal muscle during recovery from endurance exercise. FASEB J. 2005;19(11): $1498-500$

14. Mastaloudis A, Leonard SW, Traber MG. Oxidative stress in athletes during extreme endurance exercise. Free Radic Biol Med. 2001;31(7):911-22.

15. Mastaloudis A, Traber MG, Carstensen K, Widrick JJ. Antioxidants did not prevent muscle damage in response to an ultramarathon run. Med Sci Sports Exerc. 2006;38(1):72-80.

16. McGee SL, Hargreaves M. Exercise and myocyte enhancer factor 2 regulation in human skeletal muscle. Diabetes. 2004;53(5):1208-14.

17. Merksamer PI, Trusina A, Papa FR. Real-time redox measurements during endoplasmic reticulum stress reveal interlinked protein folding functions. Cell. 2008;135(5):933-47.

18. Murton AJ, Constantin D, Greenhaff PL. The involvement of the ubiquitin proteasome system in human skeletal muscle remodelling and atrophy. Biochim Biophys Acta. 2008;1782(12):730-43.

19. Nedergaard A, Vissing K, Overgaard K, Kjaer M, Schjerling P. Expression patterns of atrogenic and ubiquitin proteasome component genes with exercise: effect of different loading patterns and repeated exercise bouts. $J$ Appl Physiol. 2007;103(5):1513-22.

20. Nielsen AR, Pedersen BK. The biological roles of exerciseinduced cytokines: IL-6, IL-8, and IL-15. Appl Physiol Nutr Metab. 2007;32(5):833-9.

21. Nieman DC, Davis JM, Brown VA, et al. Influence of carbohydrate ingestion on immune changes after $2 \mathrm{~h}$ of intensive resistance training. J Appl Physiol. 2004;96(4):1292-8.

22. Nieman DC, Henson DA, Davis JM, et al. Quercetin ingestion does not alter cytokine changes in athletes competing in the Western States Endurance Run. J Interferon Cytokine Res. 2007; 27(12):1003-11.

23. Nieman DC, Henson DA, Dumke CL, et al. Ibuprofen use, endotoxemia, inflammation, and plasma cytokines during ultramarathon competition. Brain Behav Immun. 2006;20(6):578-84.
24. Ostrowski K, Rohde T, Zacho M, Asp S, Pedersen BK. Evidence that interleukin-6 is produced in human skeletal muscle during prolonged running. $J$ Physiol. 1998;508( pt 3):949-53.

25. Ozcan U, Cao Q, Yilmaz E, et al. Endoplasmic reticulum stress links obesity, insulin action, and type 2 diabetes. Science. 2004; 306(5695):457-61.

26. Papassotiriou I, Alexiou VG, Tsironi M, Skenderi K, Spanos A, Falagas ME. Severe aseptic inflammation caused by long distance running $(246 \mathrm{~km})$ does not increase procalcitonin. Eur J Clin Invest. 2008;38(4):276-9.

27. Penkowa M, Keller P, Keller C, Hidalgo J, Giralt M, Pedersen BK. Exercise-induced metallothionein expression in human skeletal muscle fibres. Exp Physiol. 2005;90(4):477-86.

28. Peter A, Weigert $\mathrm{C}$, Staiger $\mathrm{H}$, et al. Individual stearoyl-CoA desaturase 1 expression modulates endoplasmic reticulum stress and inflammation in human myotubes and is associated with skeletal muscle lipid storage and insulin sensitivity in vivo. Diabetes. 2009; 58(8):1757-65.

29. Petersen AM, Pedersen BK. The anti-inflammatory effect of exercise. J Appl Physiol. 2005;98(4):1154-62

30. Reid MB. Response of the ubiquitin-proteasome pathway to changes in muscle activity. Am J Physiol Regul Integr Comp Physiol. 2005;288(6):R1423-31.

31. Roberts CK, Barnard RJ, Sindhu RK, Jurczak M, Ehdaie A, Vaziri ND. Oxidative stress and dysregulation of NAD(P)H oxidase and antioxidant enzymes in diet-induced metabolic syndrome. Metabolism. 2006;55(7):928-34.

32. Ron D, Walter P. Signal integration in the endoplasmic reticulum unfolded protein response. Nat Rev Mol Cell Biol. 2007;8(7): 519-29.

33. Sahlin K, Shabalina IG, Mattsson CM, et al. Ultra-endurance exercise increases the production of reactive oxygen species in isolated mitochondria from human skeletal muscle. J Appl Physiol. 2010;108(4):780-7.

34. Starkie R, Ostrowski SR, Jauffred S, Febbraio M, Pedersen BK. Exercise and IL-6 infusion inhibit endotoxin-induced TNF-alpha production in humans. Faseb J. 2003;17(8):884-6.

35. van der Vlies D, Makkinje M, Jansens A, et al. Oxidation of ER resident proteins upon oxidative stress: effects of altering cellular redox/antioxidant status and implications for protein maturation. Antioxid Redox Signal. 2003;5(4):381-7.

36. Vembar SS, Brodsky JL. One step at a time: endoplasmic reticulumassociated degradation. Nat Rev Mol Cell Biol. 2008;9(12):944-57.

37. Wing SS. Control of ubiquitination in skeletal muscle wasting. Int $J$ Biochem Cell Biol. 2005;37(10):2075-87.

38. Witt SH, Granzier H, Witt CC, Labeit S. MURF-1 and MURF-2 target a specific subset of myofibrillar proteins redundantly: towards understanding MURF-dependent muscle ubiquitination. $J \mathrm{Mol} \mathrm{Biol}$. 2005;350(4):713-22.

39. Wojtaszewski JF, Nielsen P, Kiens B, Richter EA. Regulation of glycogen synthase kinase-3 in human skeletal muscle: effects of food intake and bicycle exercise. Diabetes. 2001;50(2):265-9.

40. Yoshida H, Haze K, Yanagi H, Yura T, Mori K. Identification of the cis-acting endoplasmic reticulum stress response element responsible for transcriptional induction of mammalian glucoseregulated proteins: involvement of basic leucine zipper transcription factors. J Biol Chem. 1998;273(50):33741-9. 